\title{
Los ambientes de aprendizaje como metodología activa promotora de la actividad física en Educación Infantil. Un estudio de caso Learning environments as an active methodology to promote physical activity in Early Childhood Education. A case study \\ Adriana Nielsen Rodríguez, Ángel Ramón Romance García, José Luis Chinchilla Minguet \\ Universidad de Málaga (España)
}

Resumen: La presente investigación centra su foco de interés en el estudio de caso de una práctica innovadora en Educación Infantil relacionada con el fomento de la actividad física como potenciadora del desarrollo cognitivo, concepto que representa una de las cuestiones más debatidas en la investigación educativa actual. Esto, unido al papel esencial de dicha etapa en el desarrollo y a la alta tasa de inactividad infantil, hace de nuestro tema una prioridad en numerosos países, pero para abordar esta problemática es preciso un cambio radical en los modos de enseñar y aprender. Nuestro objetivo es profundizar en el conocimiento de metodologías activas implementadas en Educación Infantil y descubrir cómo favorecen la práctica de actividad física entre el alumnado, instaurando hábitos más activos en las escuelas. Para ello estudiamos una experiencia basada en ambientes de aprendizaje desde una perspectiva analítico-descriptiva, empleando la metodología del estudio de caso junto con otras técnicas y herramientas de recogida y análisis de la información. Los resultados evidencian que esta metodología precisa de un continuo movimiento por parte del alumnado, tanto para acceder a las distintas propuestas como para realizarlas, por lo que parece oportuno considerarla como favorecedora de la actividad física y, por ende, de un óptimo desarrollo de las capacidades físicas y cognitivas de los niños y niñas.

Palabras clave: Educación Infantil, Metodología Activa, Ambientes de Aprendizaje, Actividad Física, Desarrollo Cognitivo, Funciones Ejecutivas, Innovación Educativa.

\begin{abstract}
This research focuses on a case study of an innovative educational practice in Early Childhood Education related to the promotion of physical activity as an enhancer of cognitive development, a concept that represents one of the most debated issues in current educational research. This, together with the essential role of this life period in children's development, and the high rate of inactive youth, makes our topic a priority in many countries; however, a radical change in teaching is required in order to address this topic, including the implementation of active and innovative methodologies. The objective of this paper is to deepen the knowledge of active methodologies that are being implemented in Early Childhood Education and discover how they favor the practice of physical activity among students, contributing to establish more active habits in schools. To do so, we studied an innovative experience based on learning environments, and we investigated the way in which it encourages students' physical activity from an analytical-descriptive perspective, using the case study methodology together with other techniques and tools for collecting and analyzing information. The results show that this methodology requires students to move continuously, both to access the different proposals and to perform them, so it seems appropriate to consider it as an enhancer of physical activity and, therefore, of an optimal development of children's physical and cognitive abilities.
\end{abstract}

Keywords: Early Childhood Education, Active Methodology, Learning Environments, Physical Activity, Cognitive Development, Executive Functions, Educational Innovation.

\section{Introducción}

El Neuroconstructivismo, una disciplina muy actual y ambiciosa derivada del constructivismo (Westermann, Mareschal, Johnson, Sirois, Spratling \& Thomas, 2007), tiene como objetivo entender el desarrollo de la mente humana y estudiar cuáles son las bases cerebrales que subyacen a la adquisición de las funciones cognitivas básicas, así como aquellas que se adquieren en los contextos educativos (Enríquez, 2014; Redolar, 2015), especialmente en las primeras edades. Para ello, reúne los conocimientos de la Psicología Evolutiva y los hallazgos de la Neurociencia Cognitiva en relación a los procesos de desarrollo cognitivo (Westermann et al., 2007; Sirois, Spratling, Thomas, Westermann, Mareschal \& Johnson, 2008) y postula que la mente existe encarnada en un cerebro y un cuerpo, y que el desarrollo no viene predeterminado sino que supone un proceso progresivo que da lugar a las principales funciones psicológicas (Enríquez, 2014).

Las investigaciones más recientes en el campo de la

Fecha recepción: 19-02-19. Fecha de aceptación: 04-06-19 Adriana Nielsen Rodríguez adriananielsen@uma.es neurociencia demuestran que el ejercicio físico hace que nuestro cerebro funcione de manera óptima (Ratey \& Hagerman, 2008), influye en la neurogénesis, la sinaptogénesis y la creación de conexiones neuronales en áreas cerebrales como la corteza motora, prefrontal o el hipocampo, mejorando nuestro rendimiento cognitivo (Rosa, García \& Carrillo, 2019; Maureira, Bravo, Aguilera, Bahamondes \& Véliz, 2019), y mantiene un estado cerebral saludable. En las raíces de nuestra biología encontramos signos de la influencia del cuerpo sobre la mente (MartínezLópez, 2003), así como evidencias de que el movimiento produce efectos en nuestro organismo que juegan papeles cruciales en los mecanismos relacionados con los procesos de pensamiento más elevados (Ratey \& Hagerman, 2008).

Este corpus emergente de investigación establece que las funciones cerebrales dependen de un componente motor que es imprescindible para su correcto desarrollo (Enríquez, 2014; Maureira, et al., 2019) y reconoce los efectos beneficiosos del ejercicio aeróbico sobre ciertos aspectos de la función cerebral particularmente importantes para la educación (Hillman, Erickson \& Kramer, 2008), incluyendo las funciones ejecutivas, el cociente intelectual y el rendimiento académico (Sibley \& Etnier, 2003; Ramírez, Vinaccia \& Suárez, 2004; Kovacs, Gil, Gestoso, López, Mufraggi \& Palou, 2008; 
Marques, Gómez, Martins, Catunda \& Sarmento, 2017). Además, está ampliamente demostrado que la realización de un ejercicio físico regular a intensidades aeróbicas de moderadas a vigorosas (del $40 \%$ al $80 \%$ del VO máx) actúa positivamente sobre algunas funciones cognitivas tales como la velocidad de respuesta, la memoria a corto plazo, la motivación (Etnier, Nowell, Landers \& Sibley, 2006; Hillman et al, 2008; Lambourne \& Tomporowski, 2010), la inhibición o la flexibilidad cognitiva (Rosa, et al., 2019). Sin embargo, el carácter sedentario de la vida moderna supone una alteración de nuestra naturaleza y una de las mayores amenazas para nuestra supervivencia continua (Beltrán, Sierra, Jiménez, GonzálezCutre, Martínez y Cervelló, 2017), de modo que esta inactividad no sólo está perjudicando a nuestros cuerpos, sino que está marchitando nuestros cerebros. De hecho, de acuerdo con el Eurobarómetro 2018 de la Comisión Europea, entre el $40 \%$ y el $60 \%$ de la población lleva una vida sedentaria.

Para responder a estas demandas de la sociedad actual debemos transformar la escuela de modo que pueda satisfacer las necesidades de su alumnado (Ainscow, Dyson, Goldrick \& West, 2016; Cañabate, Tesouro, Puiggale, \& Zagalaz, 2019). En este sentido, las prácticas innovadoras suponen valiosas aportaciones que persiguen la mejora de las escuelas (OCDE, 2015) y son una fuente inagotable de ideas y saberes a tener en cuenta (Escudero, 2014) para avanzar en el ámbito educativo hacia modelos de calidad y de excelencia (Cañabate, et al., 2019).

La investigación se enmarca en Educación Infantil porque supone un momento crítico del desarrollo en el que se produce un rápido crecimiento del sistema nervioso central, se establece la arquitectura cerebral (Eurydice, 2013), existen una intensa actividad neural y maduración neuromuscular y las funciones ejecutivas comienzan a desarrollarse (Tirapu, García, Ríos \& Ardila, 2012). A esto se unen las conclusiones desprendidas del Neuroconstructivismo y la Neurociencia Cognitiva, que concluyen que la práctica de ejercicio físico tiene que comenzar desde la infancia (Myer, Faigenbaum, Edwards, Clark, Best \& Sallis, 2015; Beltrán, et al., 2017), y que este enriquecimiento cognitivo obtenido en edades tempranas resulta ser protector contra el declive cognitivo asociado a la edad y también a la demencia (Redolar, 2015).

Por ello la Organización Mundial de la Salud (2010) insta a trabajar para el impulso de metodologías activas que promuevan la adquisición de hábitos relacionados con la actividad física que perduren en el tiempo, lo cual es relativamente fácil de implementar en Educación Infantil utilizando una intervención apropiada (Epstein, Valoski, Wing \& McCurley, 1990; Inge et al., 2004; Marques, et al., 2017) basada mayoritariamente en juegos y actividades no estructuradas. Y es que el uso del juego y el movimiento libre como herramientas para la práctica de actividad física es especialmente importante en niños pequeños, ya que estas actividades no estructuradas promueven el desarrollo de la función ejecutiva en la infancia (Diamond \& Lee, 2011; Duran \& Costes, 2018; Padial-Ruz, Ibáñez-Granados, Fernández, \& UbagoJiménez, 2019).

En base a lo anterior, nos centramos en dos aspectos fundamentales para la progresiva consecución de mejoras en el sistema escolar: la implementación de metodologías innovadoras y el aumento de la actividad física que realiza el alumnado (Cañabate, et al., 2019). Dicho esto, establecemos como objetivo el estudio de los ambientes de aprendizaje como práctica de innovación en Educación Infantil y su impacto en la promoción de la actividad física del el alumnado a partir del análisis de un centro en el que se trabaje en base a dicho método.

La importancia nacional e internacional de esta temática queda reflejada no sólo en el notable incremento, en las últimas dos décadas, de publicaciones científicas en revistas especializadas, sino también por la celebración de seminarios, jornadas y congresos con esta disciplina como tema de estudio y trabajo, así como por su presencia cada vez mayor en la mayoría de líneas de investigación relacionadas con la educación, convirtiendo la promoción de la actividad física en un objetivo prioritario de salud pública (Beltrán, et al., 2017).

Además en España, al igual que en la mayoría de países, las administraciones educativas reconocen la necesidad de movimiento y contemplan la adopción de medidas para incrementar la cantidad de actividad física durante la jornada escolar (Ratey \& Hagerman, 2008; Blair, 2009; Kibbe, 2011; Sousa, 2014), diseñando propuestas como la implementación de una tercera hora de Educación Física semanal en los centros dependientes del Ministerio de Educación, Cultura y Deporte, y los programas que está desarrollando de manera conjunta este Ministerio con el de Sanidad, con líneas de trabajo específicas enfocadas al fomento de la actividad física en niños. Entre ellos encontramos el Programa Perseo, el Plan Colegios Activilandia, la Campaña Movimiento Actívate o el Programa ¡Dame 10!, que consta de una serie de descansos activos de unos diez minutos para ser desarrollados en el aula por el profesor durante el horario lectivo.

De este modo, la necesidad de incrementar la actividad física se está convirtiendo en una prioridad político-social (Beltrán, et al., 2017) y la mayoría de autores coinciden en la necesidad de impulsarla desde la Educación Infantil, vislumbrando en la innovación un medio de alcanzarla. Sin embargo, a pesar de que existe una amplia panorámica de investigaciones que relacionan los efectos de la actividad física con la cognición, no existen estudios en profundidad en contextos educativos reales en Infantil (Etnier et al., 2006; Tirapu et al., 2012), siendo los trabajos que estudian directamente el impacto de las prácticas innovadoras en esta etapa sobre la actividad física muy escasos, y de ahí deviene la importancia de nuestra investigación.

No hay duda de que la puesta en marcha de una pedagogía innovadora orientada a promocionar la actividad física desde la etapa de Educación Infantil constituye una necesidad imperiosa, y es por este motivo que son necesarios estudios como el presente para construir el conocimiento desde la perspectiva de diferentes experiencias; recopilando, analizando y difundiendo datos útiles para otros investigadores y creando una plataforma de información que sirva para la mejora de los sistemas educativos.

\section{Materialy método}

Modalidad de investigación utilizada: el estudio de caso

Abordamos el objeto de estudio por medio de la recons- 
trucción de los conceptos y acciones que abarca, describiendo y comprendiendo el modo en que se crea la experiencia a través del lenguaje. Para ello se realizó un estudio de caso, ampliamente reconocido como sistema de investigación para evaluar innovaciones educativas en su propio contexto (Simons, 2011), mediante el cual se pudo profundizar en el conocimiento de una situación determinada gracias al análisis de un modelo de innovación educativa implementado en un colegio de Educación Infantil y Primaria de la provincia de Málaga.

Ya que el estudio de caso supone un estudio de lo singular y lo distintivo (Simons, 2011), nos permitió ahondar en unos hechos específicos de interés para la investigación mediante la recogida selectiva de información, cuyo análisis nos dio la oportunidad de captar y reflejar los elementos que dan significado a la situación objeto de estudio en cuestión. Además, al entrañar un procedimiento que trasciende la mera observación, valora las múltiples perspectivas de los interesados, intentando comprender mediante el análisis y la interpretación de los datos cómo piensan, sienten y actúan los agentes involucrados.

Técnicas y herramientas para la recogida y el análisis de datos

El estudio de caso tiende al uso conjunto de diferentes metodologías. El diario junto con la entrevista, la observación y el análisis de documentos son los métodos que suelen emplear para facilitar el análisis exhaustivo y la comprensión (Simons, 2011), y por ello han sido éstas las técnicas empleadas en esta investigación.

En el diario quedan preservados no sólo datos formales, objetivos y precisos de la realidad estudiada sino también las preocupaciones, decisiones, sensaciones y valoraciones del investigador (Bautista, 2011). En él hemos recogido descripciones pormenorizadas de los ambientes, su organización y de las actividades realizadas en ellos, concretando siempre la fecha, la duración y algunos objetivos antes de cada entrada, distinguiendo quién ha dicho o hecho aquello que estamos registrando para evitar posibles equívocos.

La observación se convierte en una técnica científica cuando es planificada y comprobada mediante criterios de validez y fiabilidad (Simons, 2011). Consiste en observar atentamente el fenómeno a investigar, en este caso la metodología de Educación Infantil del centro seleccionado. Nos posicionamos como observadores no participantes, buscando complementar la información del diario con datos sobre cómo y en qué medida se está favoreciendo la práctica de actividad física entre el alumnado.

La entrevista se define como una técnica de investigación por la cual se obtienen datos mediante un diálogo entre el investigador y el entrevistado, en el que existe una intencionalidad y hay una planeación determinada (Kvale, 2011). Gracias a las entrevistas formales e informales con las docentes, el alumnado y las familias hemos tenido la oportunidad de conocer mejor algunos aspectos que hubieran podido pasar inadvertidos, además de ayudarnos a evitar malinterpretaciones acerca de la realidad investigada.

Finalmente el análisis documental, para ser científico y constituirse como instrumento para la recogida de información, debe ser total y completo (Bautista, 2011), captando el sentido tanto manifiesto como latente del texto. Se realizó de una forma sistemática, objetiva, replicable y válida, indagando en documentos de diversas fuentes y naturalezas para conocer situaciones en diferentes aspectos institucionales, grupales, formales o informales, siempre que en ellos haya sido posible capturar información valiosa.

Para la interpretación de la información recopilada se planteó un análisis en progreso de los datos con el que aligerar la carga de trabajo posterior y detectar posibles lagunas de información a ser subsanadas antes de terminar el trabajo de campo. También recurrimos a la triangulación para la búsqueda de patrones de convergencia (Simons, 2011) con los que desarrollar una interpretación global de nuestro objeto de estudio, procediendo en último lugar a la reducción y categorización de los datos con el fin de simplificarlos para expresarlos de una manera sistemática, inteligible y significativa.

\section{Resultados}

La imperiosa necesidad de innovación actual deriva principalmente de nuestra obsoleta forma de entender la educación, centrada casi en exclusividad en libros de textos y prácticas reiterativas que no son capaces de satisfacer las necesidades del alumnado (Casanova, 2009). Como consecuencia, los sistemas educativos han sido testigo en las últimas décadas de múltiples intentos de cambio que han logrado producir algunos avances, aunque aún quedan numerosos retos por conseguir (Escudero, 2014), entre los cuales destaca la necesidad fomentar el movimiento físico del alumnado en las aulas aprovechando tanto la obligatoriedad de la asistencia a clase como las oportunidades metodológicas a nuestro alcance (Marques, et al., 2017). Pero para llevar a cabo un cambio lo suficientemente profundo es necesario salir del modelo de educación en el que llevamos inmersos más de dos siglos y adentrarnos en otro que responda a las necesidades de los nuevos tiempos y, más allá aún, de los alumnos y alumnas. Esto requiere de un sistema educativo que se guíe por las necesidades del contexto, que experimente e investigue, que sea creativo y proactivo (Casanova, 2009).

\section{Los ambientes de aprendizaje: una metodología acti-} va e innovadora

En nuestra sociedad, caracterizada por su extraordinario dinamismo, se enlaza progresiva y sistemáticamente la aparición de nuevos recursos con la de nuevos problemas a solucionar, lo cual requiere una búsqueda permanente de mecanismos de adaptación y cambio con los que responder a las nuevas demandas y necesidades (Cañabate, et al., 2019). Como no podía ser de otro modo, la educación se ve constantemente envuelta en estos procesos de cambio para poder responder a las nuevas situaciones que se van dando (Escudero, 2014), y es por esto que surgen nuevas metodologías como los ambientes de aprendizaje.

La principal fuente de la que se nutre el trabajo por ambientes es una de las experiencias innovadoras de Educación Infantil con mayor reconocimiento: Reggio Emilia. Esta pedagogía fomenta la participación activa del niño en el proceso de aprendizaje y defiende la experimentación e interacción con el entorno como medio para la construcción 
del conocimiento y la educación integral de la infancia (Cañal, 2002), lo cual requiere necesariamente de movimiento físico por parte del alumnado.

El aprendizaje por ambientes también encuentra en la metodología de rincones y en los talleres dos potentes antecedentes de espacios educativos acordes con la necesidad de movimiento infantil (Riera, Ferrer \& Ribas, 2014) gracias a la variedad de espacios y propuestas que implican, si bien los ambientes son más extensos y ocupan buena parte del centro al situarse en las aulas, en los pasillos y en los espacios exteriores. Así, inspirados por estas dos prácticas, los ambientes brindan a los niños la oportunidad de elegir qué actividades realizar, rompiendo la organización espacial propia de la escuela tradicional para lograr un espacio que favorezca la libertad de movimiento del alumnado.

Mientras que la mayoría de actividades que se ponen en práctica en la escuela lo hacen en un contexto artificial, ambiguo, vacío y aburrido para el alumnado, los ambientes parten de la idea de que la motivación, la manipulación activa y el movimiento son esenciales para conseguir que se produzcan aprendizajes, y para ello procuran multiplicar las posibilidades de exploración y acción del niño (Riera et al., 2014), mediante la creación de entornos de aprendizaje ricos, flexibles, descentralizados, cooperativos y autónomos, garantizándole un entorno estimulante gracias al cual experimenta, investiga, interpreta, vive el mundo, lo comprende y lo transforma dándole sentido.

Contribución de los ambientes de aprendizaje al fomento de la actividad física

La metodología basada en ambientes de aprendizaje reconoce y acepta la necesidad de movimiento, de acción y de exploración activa por parte del alumnado para optimizar su desarrollo integral y potenciar su rendimiento cognitivo. Por ello se centra en modificar el contexto de aula desarrollando estrategias que respondan a estas necesidades mediante propuestas muy abiertas y flexibles que permiten a cada alumno acceder a ellas independientemente de sus capacidades, necesidades o ritmos individuales.

Respecto a lo que hemos podido comprobar, en esta metodología cada una de las aulas (a las que se añaden otros espacios como pasillos y patios) es convertida en un ambiente preparado para una determinada propuesta educativa. Se procuran espacios definidos, estructurados, ordenados, atractivos y motivadores que permitan el libre movimiento de niños y niñas a la vez que los invitan a actuar y a experimentar, configurando lugares donde existen y se desarrollan condiciones favorables de aprendizaje en un entorno en el que es posible llevar a la práctica una amplia gama de actividades simultáneas.

El centro observado cuenta con nueve ambientes o aulas de puertas abiertas donde cada alumno, independientemente del nivel y grupo al que pertenezca, puede acudir para realizar actividades que le motiven e interesen. Cada ambiente se nombra con un color, además de estar dedicado a una temática determinada, pero todos tienen en común el carácter activo de las actividades que albergan. Encontramos el ambiente blanco o de psicomotricidad, el rosa o de construcciones, el rojo o de creatividad y manualidades, el naranja o de arte, el morado o de lectoescritura, el verde o de matemá- ticas, el azul o de naturaleza y experimentos y el amarillo o de luz; además de un ambiente exterior.

Destacamos la presencia de un ambiente de psicomotricidad y otro exterior en los que el alumnado tiene la oportunidad de realizar actividad física programada y enfocada a optimizar su desarrollo cognitivo y motor, en el primero con material estructurado específico para la práctica psicomotriz, y en el segundo con material no estructurado pero igualmente valioso como escalinatas y cuerdas para trepar en los árboles; neumáticos que pueden mover y con los que pueden crear estructuras para escalar, reptar o saltar entre otras opciones; espacios abiertos para correr; etc. Pero además, podemos comprobar que en el resto de ambientes se ofrecen actividades para cuya realización se exige al alumnado que esté en constante movimiento, sin olvidar que para cambiar de ambiente los niños y niñas han de desplazarse por el centro continuamente.

Aquí no existen los puestos escolares de mesas y sillas asignadas, sino que cada cual busca dentro de cada ambiente la propuesta que más se adecua a sus necesidades en cada momento. El constante movimiento infantil, que en otros lugares se contempla como una problemática a solucionar, aquí se concibe como una fuente de aprendizaje de gran valor, y etiquetas como la de hiperactividad han sido desterradas para dar paso a niños vivos e inquietos que tan sólo hacen lo que cualquier niño de tres a seis años debe hacer: moverse.

Respecto a los materiales que podemos hallar en cada ambiente, es importante que estos sean capaces de provocar interrogantes, experimentos y descubrimientos. Se eligen materiales lo más naturales posibles, poco estructurados y muy abiertos en cuanto a sus posibilidades, aunque también se utilicen algunos más específicos o con mayor grado de abstracción, y se muestran de una manera atractiva y siempre al alcance del alumnado para potenciar su autonomía. Con ellos lo que se busca es promover la investigación, la exploración, la manipulación y el desarrollo de la creatividad.

En lo relativo a los destinatarios, los ambientes se caracterizan por una mezcla constante de alumnos de diferentes edades, creándose normalmente grupos heterogéneos de niños y niñas de diferentes niveles que se apoyan y se instan a superarse a sí mismos a través de un proceso activo, cooperativo, progresivo y autodirigido, que apunta a encontrar significados y construir conocimientos que surgen, en la medida de lo posible, de las experiencias directas con el entorno y con el otro. Aquí cada niño/a elige donde ir y qué hacer; son los espacios, los compañeros y los materiales los que les invitan a interactuar en un juego y una exploración libres en los que cada cual va respondiendo a sus necesidades en cada momento. La máxima de esta metodología es que alumnos y alumnas construyan sus propios aprendizajes a partir del juego (tanto libre como dirigido), la experimentación y el trabajo cooperativo, apoyados por unos docentes que ceden el protagonismo a su alumnado y se centran en observarles y orientarles, velando por el respeto de las normas comunes y preparando los ambientes para que ofrezcan todo tipo de propuestas interesantes, atractivas y con un alto potencial educativo. 


\section{Discusión}

La innovación educativa que incluye conceptos neurocientíficos válidos es un fenómeno relativamente nuevo y hemos visto que los retos implicados son considerables. Cuando los educadores consideran los procesos de aprendizaje, suelen pensar más en los procesos mentales que en los neurales, aunque en el futuro podemos prever que los constructos acerca de estos procesos mentales estarán cada vez más informados por ideas neurobiológicas (Redolar, 2015) como las que aquí barajamos, las cuales muestran que la actividad física desencadena cambios biológicos que estimulan a las células cerebrales a desarrollarse y a unirse a las demás (Maureira, et al., 2019), lo cual es necesario para aprender, y cuanto más descubren los neurocientíficos sobre este proceso más claro se vuelve el hecho de que el ejercicio proporciona un estímulo incomparable, creando un entorno en el cual el cerebro está preparado y es capaz de alcanzar su máximo potencial (Ratey \& Hagerman, 2008).

Cada vez son más las investigaciones que muestran los efectos de la práctica de actividad física en el desarrollo del cerebro, en las conexiones entre las neuronas y en el apropiado equilibrio de las sustancias neuroquímicas (Rosa, et al., 2019), y los estudios a nuestro alcance demuestran los efectos beneficiosos de la actividad física sobre el desarrollo cognitivo, las funciones ejecutivas y el aprendizaje, así como la necesidad de impulsarla desde las primeras edades (Ratey \& Hagerman, 2008; Enríquez, 2014; Redolar, 2015), convirtiendo la temática en una prioridad político-social. Entendiendo estas interacciones entre el cuerpo y el cerebro seremos capaces de gestionar el proceso, manejar los problemas y propiciar un desarrollo óptimo de las habilidades cognitivas y las funciones ejecutivas.

Existen ciertos desarrollos educativos que implican la neurociencia que pueden llegar en la próxima o en las próximas décadas (Ratey \& Hagerman, 2008). Se establecerán nuevos enfoques educativos para la enseñanza y el aprendizaje en los primeros años, desarrollándose nuevas formas de entender la educación y estableciéndose nuevos enfoques curriculares en los que se preste más atención al desarrollo de ciertas funciones cognitivas específicas y de las funciones ejecutivas. Además, los descansos para hacer ejercicio se convertirán en una norma del currículo cuando la relación entre el ejercicio físico y el rendimiento académico sea más evidente y la neurociencia se convierta en un elemento de la formación profesional inicial y continua del profesorado. De este modo, se establecerá un nuevo campo de investigación neuroeducativa, junto con el desarrollo de profesionales formados tanto en educación como en las ciencias neurocognitivas, y gracias a estos esfuerzos, las ciencias de la mente y del cerebro contribuirán de forma cada vez más importante a la práctica y la planificación educativa.

No obstante, los cambios se van produciendo mediante pequeñas innovaciones que el profesorado va introduciendo en ese día a día que conforma la realidad de la educación (Cañabate, et al., 2019). Si esos cambios resultan positivos, llega un momento en que debe dejar de ser un cambio casi inconsciente, para ser intencionado, sistematizado y difundido al resto del equipo y al conjunto del centro, por si pro- cede asumirlo globalmente y plantearlo como posible innovación educativa que se implementará hasta comprobar con certeza sus buenos resultados, tal y como ha ocurrido en el centro objeto de nuestro estudio.

Este trabajo puede suponer el punto de partida de una línea de investigación que se complete con otras intervenciones posteriores y pueda cubrir ciertas limitaciones y nuevos campos de estudio, planteando diferentes perspectivas de futuro que ayudarán aún más a profundizar en un ámbito que lo necesita. Entre estas posibles líneas de acción se pueden destacar algunas como el aumento de la muestra, la introducción de nuevas características y variables, el diseño de un programa formativo para escolares y docentes que hiciera hincapié en mejorar en los déficits de las variables que se hayan detectado y la delimitación de estudios longitudinales para hacer un seguimiento de la evolución de los grupos que han formado parte del estudio.

A tenor de lo anteriormente expuesto, concretamos una serie de recomendaciones y pautas que pueden servir para ser implementadas en otros centros y así poder extrapolar esta práctica a otros contextos escolares:

- Se han de crear contextos abiertos, participativos y flexibles que permitan el movimiento del alumnado y en los que se planteen actividades ricas en estímulos, que promuevan la investigación, la exploración, la manipulación y el juego infantil.

- Los materiales han de ser lo más naturales posibles para que permitan una gran variedad de actividades y no limiten sus posibilidades.

- Las intervenciones del profesorado se han de encaminar a facilitar en lugar de dirigir las actuaciones del alumnado, acompañando al niño y apoyándole en los retos que se plantee y provocando sus respuestas sin anticiparlas.

- La evaluación debe realizarse de manera continua y utilizando varios instrumentos con el propósito de contrastar la información obtenida.

- Se requiere de una continua formación por parte del profesorado para estar al día de las políticas y prácticas innovadoras existentes con el fin de poder aplicarlas en el caso de que fuera necesario.

\section{Conclusiones}

Lo que en este trabajo se ofrece es un estudio de campo sobre un segmento de la vida de un centro educativo caracterizado por la introducción de una metodología innovadora que no sólo facilita el aprendizaje de su alumnado, sino que establece entre ellos un principio de actividad física, movimiento y juego motor favorecedor del desarrollo de las habilidades cognitivas, de las funciones ejecutivas (Padial-Ruz, et al., 2019) e incluso de la conciencia emocional (Duran \& Costes, 2018).

Para abordar su estudio se ha procedido por múltiples vías: analizando bibliografía relacionada con el tema y fuentes documentales del propio centro, a partir de entrevistas formales e informales, mediante observaciones y notas de campo y, sobre todo, mediante la realización de un estudio de caso que permitiera organizar todas esos datos y materiales. De este modo, habiendo realizado un profundo análisis 
de la relación entre innovación, actividad física y desarrollo cognitivo en Educación Infantil como ejes vertebradores del presente estudio, teniendo en cuenta los datos recopilados y habiendo llevado a cabo un exhaustivo examen de los mismos, podemos alcanzar algunas conclusiones como las que recogemos a continuación.

Tal vez la conclusión más evidente es que el porcentaje de instituciones educativas que implementan prácticas innovadoras aún no alcanza cifras significativas y los centros con iniciativas como la aquí estudiada siguen siendo raras excepciones. Por suerte, existen equipos docentes que se atreven a arriesgar introduciendo nuevas metodologías, en este caso los ambientes de aprendizaje, siguiendo los consejos y las recomendaciones emanadas de las investigaciones más recientes que advierten de la necesidad de relacionar movimiento y aprendizaje. Por este motivo ofrecen propuestas lo suficientemente abiertas y poco estructuradas como para que provoquen la actividad infantil, pero lo suficientemente ricas y variadas como para que provoquen nuevos aprendizajes.

Se han recogido numerosos datos y evidencias que prueban que el trabajo por ambientes no sólo respeta la necesidad de movimiento del alumnado, sino que cuenta con determinados elementos que la hacen una gran propulsora de la misma. En conjunto, se pretende que a través de esta experiencia innovadora los niños aprendan y desarrollen al máximo sus capacidades física y cognitivas a la luz de los datos ofrecidos por el Neuroconstructivismo y la Neurociencia Cognitiva y, si bien es cierto que cada niño puede responder de manera diferente a las propuestas de trabajo planteadas en los ambientes, también lo es que para eso está la maestra, para intentar ayudar, animar, guiar, incentivar, acoger, fomentar la curiosidad, aportar una atención personalizada, etc.

La investigación realizada ha resultado ser, como poco, muy esclarecedora con respecto a un tema que está en las agendas más actuales y ha dejado en evidencia que existe otra forma de hacer educación, que sólo es necesario desearlo, visualizarlo, creer que una escuela mejor es posible y trabajar duro para conseguirlo.

\section{Agradecimientos}

Este trabajo ha sido financiado por el Ministerio de Educación, Cultura y Deporte a través de las ayudas para la Formación de Profesorado Universitario (FPU17/01554)

\section{Referencias}

Ainscow, M.; Dyson, A.; Goldrick, S. \& West, M. (2016). Using collaborative inquiry to foster equity within school systems: opportunities and barriers. School Effectiveness and School Improvement, 27, 7-23.

Bautista, N.P. (2011). Proceso de la investigación cualitativa. Epistemología, metodología y aplicaciones. Colombia: El Manual Moderno.

Beltrán, V.J., Sierrra, A.C., Jiménez, A., González-Cutre, D., Martínez, C. \& Cervelló, E. (2017). Diferencias según género en el tiempo empleado por adolescentes en actividad sedentaria y actividad física en diferentes segmentos horarios del día (Gender differences in time spent by adolescents in sedentary and physical activity in different day segments). Retos. Nuevas tendencias en Educación Física, Deporte y Recreación, 31, 3-7.

Blair, S.N. (2009). Physical inactivity: The biggest public health problema of the 21st century. British Journal os Sports Medicine, 43(1), 1-2.

Cañabate, D., Tesouro, M., Puiggale, J. \& Zagalaz, M.L. (2019). Estado actual de la Educación Física desde el punto de vista del profesorado. Propuestas de mejora (Current state of Physical Education from the point of view of teachers. Improvement proposals). Retos. Nuevas tendencias en Educación Física, Deporte y Recreación, 35, 47-53.

Cañal, P. (coord.) (2002). La innovación educativa. Madrid: Akal.

Casanova, M.A. (2009). Diseño curricular e innovación educativa. Madrid: La Muralla.

Comision Europea (2018). Special Eurobarometer 472. Sport and Physical Activity. Sofia. http://doi.org/10.2766/ 483047

Diamond, A. \& Lee, K. (2011). Interventions shown to Aid Executive Function Development in Children 4-12 Years Old. Science, 333(6045), 959-964. http://doi.org/10.1126/ science.1204529

Duran, C. \& Costes, A. (2018). Efecto de los juegos motores sobre la toma de conciencia emocional (Effect of Motor Games on Emotional Awareness). Revista Internacional de Medicina y Ciencias de la Actividad Física y el Deporte, 18(70), 227-245. http://dx.doi.org/10.15366/ rimcafd2018.70.003

Enríquez, P. (coord.) (2014). Neurociencia cognitiva. Madrid: Sanz y Torres.

Epstein, L.H., Valoski, A., Wing, R.R., \& McCurley, J. (1990). Ten-year follow-up of behavioral, family-based treatment for obese children. Jama, 264(19), 2519-2523. http:// doi.org/10.1001/jama.1990.03450190051027

Escudero, J.M. (2014). Avances y retos en la promoción de la innovación en los centros educativos. Educar especial 30 aniversario, 101-138.

Etnier, J.L., Nowell, P.M., Landers, D.M. \& Sibley, B.A. (2006). A meta-regression to examine the relationship between aerobic fitness and cognitive performance. Brain Research Reviews, 52, 119-130. http://doi.org/10.1016/ j.brainresrev.2006.01.002

Eurydice (2013). Key Data on Teachersad School Leaders in European. Luxembourg: Publications Office of the European Union.

Hillman, C.H., Erikson, K.I. \& kramer, A.F. (2008). Be smart, exercise your heart: Exercise effects on brain and cognition. Nature Reviews Neuroscience, 9(1), 58-65. http://doi.org/10.1038/nrn2298

Inge, T.H., Krebs, N.F., Garcia, V.F., Skelton, J.A., Guice, K.S., Strauss, R.S., ... Daniels, S.R. (2004). Bariatric surgery for severely overweight adolescents: Concerns and recommendations. Pediatrics, 114(1), 217-223.

Kibbe, D.L., Hackett, J., Hurley, M., McFarland, A., Schubert, K.G., Schultz, A. \& Harris, S. (2011). Ten years of TAKE 10 !: integrating physical activity with academic concepts in elementary school classrooms. Preventive Medicine, 52(Suppl1), S43-S50. http://doi.org/10.1016/ 
j.ypmed.2011.01.025

Kovacs, F.M., Gil, M.T., Gestoso, M., López, J., Mufraggi, N. \& Palou, P. (2008). Association between lifestyle habits and academic grades in adolescents. Apunts. Medicina De l'Esport, 43(160), 165-204.

Kvale, S. (2011). Las entrevistas en investigación cualitativa. Madrid: Morata.

Lambourne, K. \& Tomporowski, P.D. (2010). The effect of acute exercise-induced arousal on cognitive task performance: a meta-regression analysis. Brain Research Reviews, 1341, 12-24. http://doi.org/10.1016/ j.brainres.2010.03.091

Marques, A., Gómez, F., Martins, J., Caunda, R. \& Sarmento, H. (2017). Association between physical education, school-based physical activity, and academic performance: a systematic review (Asociación entre la educación física, la actividad física en la escuela, y el rendimiento académico: una revisión sistemática). Retos. Nuevas tendencias en Educación Física, Deporte y Recreación, 31,316-320.

Martínez-López, E.J. (2003). Aplicación de la prueba de lanzamiento de balón medicinal, abdominales superiores y salto horizontal a pies juntos. Resultados y análisis estadístico en Educación Secundaria. Revista Internacional de Medicina y Ciencias de la Actividad Física y el Deporte, 3(12), 223-241.

Maureira, F., Bravo, P., Aguilera, N., Bahamondes, V. \& Véliz, C. (2019). Relación de la composición corporal, las cualidades físicas y funciones cognitivas en estudiantes de educación física (Relation between body composition, physical qualities, and cognitive function in students of physical education). Retos. Nuevas tendencias en Educación Física, Deporte y Recreación, 36, 103-106.

Myer, G.D., Faigenbaum, A.D., Edwards, N.M., Clark, J.F., Best, T.M. \& Sallis, R.E. (2015). Sixty minutes of what? A developing brain perspective for activating children with an integrative exercise approach. British Journal of Sports Medicine, 49(23), 1510-1516. http://doi.org/ 10.1136/bjsports-2014-093661

OCDE (2015). Equity and Quality in Education. Supporting Disadvantaged Students and Schools. OECD.

Organización Mundial de la Salud (2010). Recomendaciones mundiales sobre actividad física para la salud. Recuperado de: http://www.who.int/dietphysicalactivity/ publications/9789241599979/es/
Padial-Ruz, R., Ibáñez-Granados, D., Fernández, M. \& UbagoJiménez, J.L. (2019). Proyecto de baile flamenco: desarrollo motriz y emocional en educación infantil (Flamenco dance project: motor and emotional development in early childhood education). Retos. Nuevas tendencias en Educación Física, Deporte y Recreación, 35, 396-401.

Ramírez, W., Vinaccia, S. \& Suárez, G.R. (2004). El impacto de la actividad física y el deporte sobre la salud, la cognición, la socialización y el rendimiento académico: Una revisión teórica. Revista De Estudios Sociales, 18, 6775.

Ratey, J. \& Hagerman, E. (2008). Spark: The revolutionary new science of exercise and the brain. New York: Little, Brown and Company.

Redolar, D. (coord.) (2015). Neurociencia cognitiva. Madrid: Editorial Médica Panamericana.

Riera, M.A.; Ferrer, M. \& Ribas, C. (2014). La organización del espacio por ambientes de aprendizaje en la Educación Infantil: significados, antecedentes y reflexiones. Revista Latinoamericana de Educación Infantil, 3(2), 19-39.

Rosa,A., García, E. \& Carrillo, P.J. (2019). Capacidad aeróbica y rendimiento académico es escolares de educación primaria (Aerobic capacity and academic performance in primary schoolchildren). Retos. Nuevas tendencias en Educación Física, Deporte y Recreación, 35, 351-354.

Sibley, B.A. \& Etnier, J.L. (2003). The relationship between physical activity and cognition in children: A metaanalysis. Pediatric Exercise Science, 15(3), 243-256. http://doi.org/10.1123/pes.15.3.243

Simons, H. (2011). El estudio de caso: teoría y práctica. Madrid: Morata.

Sirois, S., Spratling, M., Thomas, M.S., Westermann, G., Mareschal, D. \& Johnson, M.H. (2008). Précis of neuroconstructivism: how the brain constructs cognition. Behavioral and Brain Sciences, 31(3), 321-31. http:// doi.org/10.1017/S0140525X0800407X

Sousa, D. (2014). Neurociencia educativa. Mente, cerebro y educación. Madrid: Narcea.

Tirapu, J., García, A., Ríos, M. \& Ardila, A. (2012). Neuropsicología de la corteza prefontral y las funciones ejecutivas. Barcelona: Viguera Editores.

Westermann, G., Mareschal, D., Johnson, M.H., Sirois, S., Spratling, M.W. \& Thomas, M.S. (2007). Neuroconstructivism. Developmental Science, 10(1), 7583. http://doi.org/10.1111/j.1467-7687.2007.00567.x

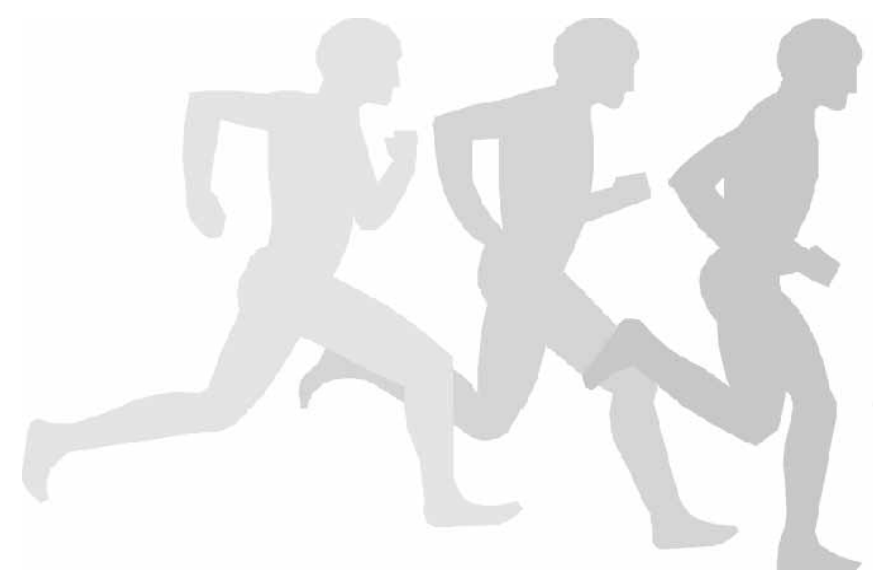

